\title{
Paths of charges in general relativity as geodesics of Einstein's non-Riemannian geometry
}

\author{
R. R. Burman
}

\begin{abstract}
The equation of motion of charged incoherent matter, and hence of a test charge, in general relativity can be written as the geodesic equation of an affine connection. Here, the connection is chosen to satisfy a condition which has the form of one of the basic geometrical principles of Einstein's unified field theory. The symetric part of the fundamental tensor of the geometry is chosen to be the metric tensor of general relativity; equations to be satisfied by the skew part, involving the electromagnetic field, are obtained. An. alternative condition on the affinity is also considered.
\end{abstract}

\section{Introduction}

In general relativity, matter can be described by an energy-momentum tensor, or by singularities in the field with the empty space field equations applicable outside the singularities [1]. With the former description, it is easily shown that the equations of motion of incoherent matter (dust) follow from the field equations; in the absence of non-gravitational fields, the particles follow geodesics of the Riemannian space of general relativity. In particular, this result applies to a single test particle, as is seen by taking the density to be proportional to a delta function $[15 ; 16$, p. 20]. Einstein, Infeld and Hoffmann derived the equations of motion of gravitating particles in their total

Received 31 August 1970. 
gravitational field by using the second description.

In general relativity, the equations of motion of a test charge in an electromagnetic field can be written as the equations of geodesics in a Finsler space or in a five dimensional Riemannian space [16, p. 35]. They can also be expressed, by suitably choosing an affine connection, in the form of the geodesic equations of that connection [4]; the affinity is the sum of the usual Christoffel symbol and a third-rank tensor which depends on the electromagnetic field. The affinity discussed by Droz-Vincent [4] is not unique, there being a class of affinities having the same geodesics, but was chosen so that the covariant derivative of the metric tensor, taken with respect to the affinity, would vanish. In the present paper, a different condition is imposed on the affinity; this condition, which involves a non-symmetric second rank tensor generalizing the metric tensor, arises as one of the basic principles of the non-symmetric unified field theory developed by Einstein, Schrödinger and others [5, 7, 13, 14].

\section{Basic theory}

\subsection{Equations of motion}

In general relativity, the path of a charged incoherent fluid in the presence of an electromagnetic field $\left(\phi_{\mu \nu}\right)$ can be represented by (see the Appendix)

$$
u^{\nu} u_{; \nu}^{\mu}=-\alpha \phi^{\alpha \mu_{u}}
$$

where $\left(u^{\mu}\right)$ is the 4-velocity of the fluid and $a$ is defined in the Appendix. Also, a semi-colon denotes covariant differentiation with respect to the usual Christoffel symbols, and the summation convention applies. Equation (1) can be written as the geodesic equation of an affine connection:

$$
u^{\nu} u^{\mu} \cdot v=0
$$

where a dot denotes covariant differentiation with respect to the affinity $\Delta$ which has components [4] 


$$
\Delta_{\alpha \beta}^{\mu}=\left\{\begin{array}{c}
\mu \\
\alpha \beta
\end{array}\right\}+a \phi_{\alpha}^{\mu} u_{\beta}
$$

The braces denote the usual Christoffel symbol formed from the metric tensor $\left(g_{\mu \nu}\right)$ of the Riemannian space of general relativity.

The above connection is not unique, but has been chosen to give $g_{\mu \nu . \rho}=0$. If $\left(V_{\alpha}\right)$ is a vector and $\left(B_{\alpha \beta}^{\mu}\right)$ an anti-symmetric third rank tensor, the quantities

$$
D_{\alpha \beta}^{\mu}=\Delta_{\alpha \beta}^{\mu}+\delta_{\alpha \beta}^{\mu} V_{\beta}+\delta_{\beta}^{\mu} V_{\alpha}+B_{\alpha \beta}^{\mu}
$$

form a connection with the same geodesics as $\Delta$. If $V_{\alpha}=0$ and the torsion vector of $B$ vanishes, $g_{\mu \nu . \rho}=0$ holds with respect to $D$ if it does with respect to $\Delta$ [4]. The geodesics of $\Delta$ are unaffected by its anti-symetric part, but for $g_{\mu v . p}=0$ to hold, $\Delta$ must be non-symmetric: a tensor $B$ chosen to cancel the skew part of $\Delta$ would have a non-zero torsion vector.

\subsection{Einsteinian geometry}

Let $\left(g_{\mu \nu}\right)$ now be some non-symmetric tensor field and let a dot denote covariant differentiation with respect to a non-symmetric affinity $\Gamma$. Compare the equation $g_{\mu \nu . \rho}=0$, which can be written

$$
g_{\mu \nu, \rho}=g_{\alpha \nu} \Gamma_{\mu \rho}^{\alpha}+g_{\mu \alpha} \Gamma_{\nu \rho}^{\alpha}
$$

where a comma denotes partial differentiation, with

$$
g_{\mu \nu, \rho}=g_{\alpha \nu} \Gamma_{\mu \rho}^{\alpha}+g_{\mu \alpha \rho \nu} \Gamma_{\rho}^{\alpha}
$$

Equation (6) differs from (5) through the different order of the indices $\nu$ and $\rho$ in the last term. Equation (6), a basic geometrical principle of the unified field theory developed by Einstein and others, has been studied in great detail by Hlavafý [8-11]; also, other authors have obtained solutions in various forms $[2,6,17,18]$.

Round and square brackets around indices denote the symmetric and 
skew-symetric parts, taken, when there are more than two indices enclosed, with respect to those immediately inside the brackets. Write $h_{\mu \nu}$ for $g_{(\mu \nu)}$ and $k_{\mu \nu}$ for $g_{[\mu \nu]}$. Let indices other than those of $g_{\mu \nu}$ be raised and lowered by using $h_{\mu \nu}$ and $h^{\mu \nu}$, where the covariant components $h^{\mu \nu}$ are defined by [8]

$$
h^{\lambda v_{\lambda \mu}}=\delta_{\mu}^{\nu}
$$

The contravariant components $g^{\mu \nu}$ of the fundamental tensor are defined by

$$
g_{\lambda \mu} g^{\lambda \nu}=g_{\mu \lambda} g^{\mu \nu}=\delta_{\lambda}^{\nu}
$$

Consider an entity $\Gamma$ with components written in the form

$$
\Gamma_{\alpha \beta}^{\mu}=\left\{\begin{array}{c}
\mu \\
\alpha \beta \beta
\end{array}\right\}+U_{\alpha \beta}^{\mu}+S_{\alpha \beta}^{\mu}
$$

where the Christoffel symbols are defined in terms of $\left(h_{\mu \nu}\right), S$ is the torsion tensor of $\Gamma$, given by

$$
S_{\alpha \beta}^{\mu} \equiv \Gamma_{[\alpha \beta]}^{\mu} \equiv \frac{1}{2}\left(\Gamma_{\alpha \beta}^{\mu}-\Gamma_{\beta}^{\mu}{ }_{\alpha}^{\mu}\right) \text {, }
$$

while

$$
\begin{aligned}
U_{\alpha \beta}^{\mu} & \equiv 2 h^{\mu \lambda} S_{\lambda(\alpha} v_{\beta} k_{\beta} \nu \\
& =2 S^{\mu}\left(\alpha{ }^{\left.v_{k}\right) \nu} .\right.
\end{aligned}
$$

It has been shown [11, p. 52] that if. (6) has a solution $\Gamma$, it must be of the form described by (9), (10) and (11). This theorem will be used in the next section.

Let

$$
K_{\omega \mu \nu} \equiv g_{\nu \mu ; \omega}+g_{\omega \nu ; \mu}+g_{\omega \mu ; \nu} \cdot
$$

Hlavatý [8, Theorem 2.3] showed that the tensor $S$ satisfies 


$$
2 S_{\omega \mu \nu}=K_{\omega \mu \nu}-4 U_{\alpha \nu[\mu} k_{\omega]}^{\alpha},
$$

$U$ being given by (11).

It was shown $[11, \mathrm{p} .58]$ that the requirements for the existence and uniqueness of a solution of (6) do not impose any restriction in the form of an equation on the components $g_{\mu \nu}$. A necessary condition for (5) to have a solution is that $[11, \mathrm{p} .48] \mathrm{g} / \mathrm{h}$ and $k / h$ must be constant, where $g, h$ and $k$ are the determinants of $\left(g_{\mu \nu}\right),\left(h_{\mu \nu}\right)$ and $\left(k_{\mu \nu}\right)$. Thus, the condition (6) produces a more flexible theory than (5).

\section{The new affinity}

Since the geodesics of an affinity are unaffected by a transformation of the form (4), it is of interest to attempt to find an affinity $D$, related by such a transformation to $\Delta$, which will satisfy a condition of the type (6).

The symmetric part of $\left(g_{\mu \nu}\right)$, namely $\left(h_{\mu \nu}\right)$, is chosen to be the Riemannian metric tensor of general relativity, so the Christoffel symbols in (3) and (9) are the same. To retain the same geodesics, the symmetric part of the new affinity must differ from that of $\Delta$ by only the terms involving $\left(V_{\lambda}\right)$ in (4). Thus, $D$ can be written in the form (9) with

$$
U_{\alpha \beta}^{\mu}=\alpha \phi\left(\alpha{ }^{\mu} u_{B}+2 \delta^{\mu}\left(\alpha V_{B}\right)\right.
$$

and with some suitable skew-symmetric tensor chosen for $\left(S_{\alpha \beta}{ }^{\mu}\right)$. Taking

$$
S_{\alpha \beta}^{\mu}=-\frac{a}{2} \phi_{\alpha \beta} L^{\mu}
$$

where $\left(L^{\mu}\right)$ is some vector, (11) and (14) give

$$
\left.\left.a \phi_{(\alpha \mu}\left(L^{\nu_{k}} k_{\beta}-u_{\beta}\right)\right)=2 h_{\mu(\alpha} V_{\beta}\right) .
$$

If $V_{\beta}$ is put equal to zero, (16) is satisfied if 


$$
k_{B \nu} L^{\nu}=u_{B}
$$

If $\operatorname{det}\left(k_{\alpha \beta}\right) \neq 0,(17)$ implies that

$$
L^{\mu}=\vec{k}^{\mu \sigma} u_{\sigma}
$$

where $\left(k^{\mu \nu}\right)$ is defined by

$$
\vec{k}^{\alpha \sigma_{\alpha \beta}}=\delta_{\beta}^{\alpha}
$$

With $V^{\mu}=0$ and $L^{\mu}$ given by (18), equations (14) and (15) become

$$
U_{\alpha \beta}^{\mu}=a \phi\left(\alpha{ }^{\mu} u_{\beta}\right)
$$

and

$$
S_{\alpha \beta}{ }^{\mu}=-\frac{a}{2} \phi_{\alpha \beta} \bar{k}^{\mu \sigma} u_{\sigma}
$$

So

$$
\left.D_{\alpha \beta}^{\mu}=\left\{\begin{array}{c}
\mu \\
\alpha \beta \beta
\end{array}\right\}+\alpha \phi_{(\alpha}{ }^{\mu} u_{\beta}\right)-\frac{\alpha}{2} \phi_{\alpha \beta} \bar{k}^{\mu \sigma} u_{\sigma}
$$

This has the same symmetric part as $\Delta$; only the torsion is different, and that does not affect the geodesics. The torsion vector of $D$ is given by

$$
D_{\alpha} \equiv D_{[\alpha \tau]}^{\tau}=S_{\alpha \tau}^{\tau}=-\frac{\alpha}{2} \phi_{\alpha \tau} \bar{k}^{\tau \sigma} u_{\sigma} .
$$

The skew-symmetric part of $\left(g_{\mu v}\right)$ has not yet been specified.

Maxwell's equations are

$$
\phi_{; \beta}^{\alpha \beta}=\frac{4 \pi}{c} \overline{\rho u}^{\alpha}
$$

and

$$
\phi_{\mu \nu, \rho}+\phi_{\rho \mu, \nu}+\phi_{\nu \rho, \mu}=0 \text {, }
$$

$\bar{p}$ being the proper charge density. Equation (24) can be used to eliminate $u$ from (21), (22) and (23). 


\section{The skew part of the fundamental tensor}

Substituting (20) and (21) into (13) results in

(26) $k_{\nu \mu ; \omega}+k_{\omega \nu ; \mu}+k_{\omega \mu ; \nu}=2 \alpha\left(\phi_{v \alpha} u_{[\mu}+\phi_{[\mu \alpha} u_{\nu}\right) k_{\omega]}^{\alpha}-a \phi_{\omega \mu} \bar{k}_{\nu}^{\sigma} u_{\sigma}$. An alternative condition can be obtained as follows.

Equation (6) can be written [8, Theorem 2.1a]

$$
g_{\mu \nu, \rho}=2 g_{\mu \alpha} S_{\rho \nu}^{\alpha}
$$

From the expression for a covariant derivative with respect to an affinity expressed in the form (9), it follows that

$$
g_{\mu \nu . \rho}=g_{\mu \nu ; \rho}-g_{\alpha \nu}\left(U_{\mu \rho}^{\alpha}+S_{\mu \rho}^{\alpha}\right)-g_{\mu \alpha}\left(U_{\nu \rho}^{\alpha}+S_{\nu \rho}^{\alpha}\right) \text {. }
$$

Hence, since $h_{\mu \nu ; \rho}=0$,

(29) $g_{\mu \nu, \rho}=k_{\mu \nu ; \rho}-2 U_{(\nu \mu) \rho}-2 S_{(\mu \rho \nu)}+2 k_{[\nu \alpha}\left(U_{\mu] \rho}^{\alpha}+S_{\mu] \rho}^{\alpha}\right)$.

Substituting (29) into (27) and separating the result into its symmetric and skew-symetric parts gives

$$
\left.U_{(\nu \mu) \rho}=-k_{(\mu \alpha} \alpha_{\rho \nu}\right)^{\alpha}
$$

and

$$
k_{\mu \nu ; \rho}+2 k_{[v \alpha} U_{\mu] \rho}^{\alpha}=2 S_{\rho[\nu \mu]} .
$$

Cyclically interchanging the indices $\mu, \nu$ and $\rho$ in (3I) and summing the three equations:

$$
k_{\mu \nu, \rho}+k_{\nu \rho, \mu}+k_{\rho \mu, \nu}=-2\left(S_{\mu \nu \rho}+S_{\nu \rho \mu}+S_{\rho \mu \nu}\right)
$$

which was obtained by Hlavatý $[8$, Theorem $7.4 ; 11$, p. 61].

The theorem connecting $U$ and $S$, namely (11), can be written

$$
U_{v \mu \rho}=2 S_{v(\mu}{ }^{\alpha} k_{\rho) \alpha}
$$

taking the symmetric part with respect to $\mu$ and $v$ gives (30).

Substituting (20) and (2l) into (31) gives 


$$
k_{\mu \nu ; \rho}+a k_{[\nu \alpha}\left(\phi_{\mu]}{ }^{\alpha} u_{\rho}+\phi_{\rho}^{\alpha} u_{\mu]}\right)+a \phi_{\rho[\nu} \bar{k}_{\mu]}^{o} u_{\sigma},
$$

while substituting (21) into (32) gives

$$
k_{\mu \nu, \rho}+k_{\rho \mu, \nu}+k_{\nu \rho, \mu}=a\left(\phi_{\mu \nu} \bar{k}_{\rho}^{\sigma}+\phi_{\rho \mu} \bar{k}_{\nu}^{\sigma}+\phi_{\nu \rho} \bar{k}_{\mu}^{\sigma}\right) u_{\sigma} \text {. }
$$

Equation (24) can be used to eliminate $\mu_{\sigma}$ from (35).

Comparison with Maxwell's equations shows that $\left(k_{\mu \nu}\right)$ is not to be identified with the electromagnetic field tensor $\left(\phi_{\mu v}\right)$.

\section{The energy-momentum tensors}

Consider the tensor with components given by

$$
A_{\alpha \beta}^{\mu}=a \phi_{\alpha}^{\mu} u_{\beta}
$$

- the symmetric part of this is $U$, as given by (20). Two further tensors are now defined:

$$
I_{\mu}^{\nu} \equiv \frac{1}{2} A_{\mu \alpha \beta} A^{\alpha \nu \beta}=\frac{1}{2} a^{2} c^{2} \phi_{\mu \alpha} \phi^{\alpha \nu}
$$

and

$$
J_{\mu \nu} \equiv A_{\alpha \beta \mu} A_{\nu}^{B \alpha}=a^{2} \phi_{\alpha \beta} \phi^{B \alpha} u_{\mu} u_{\nu}
$$

The electromagnetic energy-momentum tensor has components $[16, \mathrm{p} .8]$

$$
S_{\mu}^{\nu}=\frac{1}{4 \pi}\left(\phi_{\mu \alpha} \phi^{\alpha \nu}+\frac{2}{4} \delta_{\mu}^{\nu} \phi_{\sigma \tau} \phi^{\sigma \tau}\right)
$$

which can be expressed, using (37), by [3]

$$
S_{\mu}^{\nu}=\frac{1}{2 \pi a^{2} c^{2}}\left(I_{\mu}^{\nu}-\frac{1}{4} \delta_{\mu}^{\nu} I_{\alpha}^{\alpha}\right) \text {. }
$$

The components of the matter tensor can be expressed by

$$
M_{\mu \nu}=\rho u_{\mu \nu} u_{\nu}=\rho c^{2} J_{\mu \nu} / J_{\alpha}^{\alpha},
$$

provided $J_{\alpha}^{\alpha}$ does not vanish.

Equations (40) and (41) express the energy-momentum tensors in terms 
of tensors which can be thought of as geometrical quantities, closely related to the affinity. Droz-Vincent [4] expressed $M_{\mu \nu}$ and $S_{\mu \nu}$ in terms of geometrical quantities by using both the affinity (3) and a "dual affinity".

The invariant $J_{\alpha}^{\alpha}$ is proportional to $E^{2}-H^{2}$, where $E$ and $H$ are the electric and magnetic fields. The other electromagnetic invariant can be expressed in terms of the tensor given by (36):

$$
\begin{aligned}
\frac{1}{2} \eta_{\mu \nu \sigma \rho} A_{\lambda}^{\mu \nu \lambda} A_{\lambda}^{\sigma \rho} & =\frac{1}{2} a^{2} c^{2} \eta_{\mu \nu \sigma \rho} \phi^{\mu \nu} \phi^{\sigma \rho} \\
& =a^{2} c^{2 *} \phi_{\mu \nu} \phi^{\mu \nu}
\end{aligned}
$$

where $\eta_{\mu \nu \sigma \rho}$ is the permutation tensor and $\left({ }^{*} \phi_{\mu \nu}\right)$ is the dual of $\left(\phi_{\mu v}\right)$. This invariant is proportional to E.H.

$$
\text { 6. The condition } g_{\mu \nu . \rho}=0
$$

Droz-Vincent chose an affinity to satisfy the condition (5), namely $g_{\mu \nu . \rho}=0$, with $\left(g_{\mu \nu}\right)$ taken to be the Riemannian metric tensor of general relativity. This section deals with affinities which satisfy (5) with $\left(g_{\mu v}\right)$ a non-symetric tensor field.

If the components of the new affinity are written in the form (9), then it has been shown by Hlavatý [8, Theorem 16.1] that

$$
U_{\alpha \beta}^{\mu}=-2 S_{(\alpha \beta)}^{\mu},
$$

and that

$$
2 S_{\omega \mu}^{\alpha} k_{\nu \alpha}=-K_{\omega \mu \nu}-4 U_{\nu[\omega}^{\alpha} k_{\mu] \alpha}
$$

in which $K_{\omega \mu \nu}$ is given by (12).

Let $D$ now represent an affinity, related to the affinity $\Delta$ of Section 2 by a transformation of the form (4), and so having the same geodesics as $\Delta$; further, let $D$ satisfy (5) for some non-symmetric tensor field $\left(g_{\mu \nu}\right)$, and let $\left(h_{\mu \nu}\right)$ be the metric tensor of general 
relativity.

The affinity $D$ can be written in the form (9) with $U_{\alpha \beta}^{\mu}$ given by (14) and with some suitable skew-symmetric tensor chosen for $\left(S_{\alpha \beta}^{\mu}\right)$. Taking (15) for $S_{\alpha \beta}^{\mu},(14)$ and (43) give

$$
\left.\left.a \phi_{(\alpha \mu}\left(u_{\beta}\right)+L_{\beta}\right)=-2 h_{(\alpha}^{\mu} V_{\beta}\right) .
$$

This is satisfied by $V_{\alpha}=0$ and $L_{\alpha}=-u_{\alpha}$; then (14) and (15) become

$$
U_{\alpha \beta}^{\mu}=a \phi\left(\alpha^{\mu} u_{B}\right)
$$

and

$$
S_{\alpha \beta}^{\mu}=\frac{a}{2} \phi_{\alpha \beta} u^{\mu}
$$

so that

$$
\left.D_{\alpha \beta}^{\mu}=\left\{\begin{array}{c}
\mu \\
\alpha \beta
\end{array}\right\}+a \phi_{(\alpha}{ }^{\mu} u_{\beta}\right)+\frac{a}{2} \phi_{\alpha \beta} u^{\mu} \text {. }
$$

This has the same symmetric part as $\Delta$, and so has the same geodesics. The torsion vector is given by

$$
D_{\alpha}=S_{\alpha \tau}{ }^{\tau}=\frac{a}{2} \phi_{\alpha \tau} u^{\tau}
$$

and is thus proportional to the 4 -force per unit proper volume.

The tensor $\left(k_{\mu \nu}\right)$ has not yet been specified. Since $h_{\alpha \beta ; \nu}=0$, substitution of $(46)$ and $(47)$ into $(44)$ shows that $\left(k_{\mu v}\right)$ must satisfy (50) $k_{v \mu ; \omega}+k_{\omega \nu ; \mu}+k_{\omega \mu ; \nu}+a \phi_{\omega \mu} u^{\alpha} k_{v \alpha}+2 a\left(\phi_{v}^{\alpha} u_{[\omega}+\phi_{[\omega} \alpha_{v}\right) k_{\mu] \alpha}=0$. An alternative condition can be obtained as follows.

Equation (29) gives $g_{\mu \nu . \rho}$ for an affinity written in the form (9); hence the symmetric and skew-syrametric parts of the equation $g_{\mu \nu . \rho}=0$ are

$$
U_{(\mu \nu)_{\rho}}=-S(\mu \rho \nu)
$$


and

$$
k_{\mu \nu ; \rho}+2 k_{[v \alpha}\left(U_{\mu] \rho}^{\alpha}+S_{\mu] \rho}^{\alpha}\right)=0
$$

Cyclically interchanging the indices $\mu, \nu$ and $\rho$ in (52) and summing the three equations:

$$
k_{\mu \nu, \rho}+k_{\nu \rho, \mu}+k_{\rho \mu, \nu}=2\left(k_{\mu \alpha} S_{\nu \rho}^{\alpha}+k_{\nu \alpha} S_{\rho \mu}^{\alpha}+k_{\rho \alpha} S_{\mu \nu}^{\alpha}\right) .
$$

The theorem connecting $U$ and $S$, namely (43), can be written

$$
U_{\mu \nu \rho}=-2 S_{\mu(\nu \rho)} \text {; }
$$

taking the symmetric part with respect to $\mu$ and $v$ gives (51).

Substituting (46) and (47) into (52) gives

$$
k_{\mu \nu ; \rho}+a k_{[v \alpha}\left(\phi_{\mu]}^{\alpha} u_{\rho}+\phi_{\rho}^{\alpha} u_{\mu]}+\phi_{\mu] \rho} u^{\alpha}\right)=0 \text {, }
$$

while substituting (47) into (53) gives

$$
k_{\mu \nu, \rho}+k_{\nu \rho, \mu}+k_{\rho \mu, \nu}=a\left(k_{\mu \alpha} \phi_{\nu \rho}+k_{v \alpha} \phi_{\rho \mu}+k_{\rho \alpha} \phi_{\mu \nu}\right) u^{\alpha} .
$$

Comparison with Maxwell's equations shows that $\left(k_{\mu v}\right)$ is not to be identified with the electromagnetic field tensor $\left(\phi_{\mu \nu}\right)$.

Another possibility is to retain $\Delta$ as the affinity, so that

$$
U_{\alpha \beta}^{\mu}=a \phi\left(\alpha{ }^{\mu} u_{B}\right)
$$

and

$$
S_{\alpha \beta}^{\mu}=\alpha \phi_{[\alpha}{ }^{\mu} u_{B]}
$$

these satisfy (43). Equations (44), (52) and (53) become

(59) $k_{\nu \mu ; \omega}+k_{\omega \nu ; \mu}+k_{\omega \mu ; \nu}=2 a\left(\phi_{[\mu}{ }^{\alpha_{k}}{ }_{\nu] \alpha} u_{\omega}+\phi_{[\nu}{ }^{\alpha} k_{\omega] \alpha} u_{\mu}+\dot{\phi}_{[\mu}{ }^{\alpha} k_{\omega] \alpha} u_{v}\right)$,

$$
k_{\mu \nu ; \rho}=2 a k_{[\mu \alpha} \phi_{\nu]}{ }^{\alpha} u_{\rho}
$$

and 
(61) $k_{\mu \nu, \rho}+k_{\nu \rho, \mu}+k_{\rho \mu, \nu}=2 a\left(k_{[\mu \alpha} \phi_{\nu]}{ }^{\alpha} u_{\rho}+k_{[v \alpha \rho]}^{\phi}{ }^{\alpha} u_{\mu}+k_{[\rho \alpha} \phi_{\mu]}{ }^{\alpha} u_{\nu}\right)$. If $k_{\mu \nu}$ is assumed to be proportional to $\phi_{\mu \nu}$, the right sides of (59), (60) and (61) vanish; in particular, (60) and (61) then give Maxwell's equations for charge-free regions.

\section{Concluding remarks}

In this paper, the equations of motion of charged incoherent matter, and hence of test charges, in general relativity, have been expressed as the geodesic equations of an affine connection, with the connection chosen to satisfy a basic geometrical principle of Einstein's non-symmetric unified field theory. The affinity is the sum of the usual Christoffel symbol and parts which depend on the electromagnetic field. For a test charge, the affinity depends on its charge to mass ratio and its 4-velocity: it is not an "external" property of space, independent of the particle.

Hlavatý classified the space-time, or either of the tensors $\left(g_{\mu \nu}\right)$ and $\left(k_{\mu v}\right)$, as being of the first, second or third class according as none, two or all four of the eigenvalues of $\left(k_{\mu}{ }^{v}\right)$ vanish. For a given fundamental tensor $\left(g_{\mu \nu}\right),(6)$ can have no solutions, a unique solution or more than one solution. In the four dimensional case with $\left(g_{\mu \nu}\right)$ real and $h_{\mu \nu}$ having the signature $(+++-)$, necessary and sufficient conditions for the existence and uniqueness of a solution to (6) are [11, 12] $G \neq 0$ and $G(G-2) \neq 0$ for the first and second classes, respectively, where $G$ represents the determinant of $\left(g_{\mu v}\right)$ divided by that of $\left(h_{\mu \nu}\right)$; for the third class, there is always a unique solution.

Hlavaty [8-11] solved (6) to obtain $\Gamma$ in tensorial form in terms of the components $g_{\mu \nu}$ and their derivatives, for all three classes including the degenerate cases. His solutions have not been used here, but could be useful in further investigations of the topic of this paper. 


\section{Appendix. The equations of motion}

Consider a four dimensional Riemannian space with coordinates $x^{\alpha}$ and interval $d s$ given by $d s^{2}=g_{\mu \nu} d x^{\mu} d x^{\nu},\left(g_{\mu \nu}\right)$ being the metric tensor. The contracted curvature tensor and the curvature scalar are written $\left(R_{\mu \nu}\right)$ and $R$. Covariant differentiation is denoted by a semi-colon. The field equations of general relativity are

$$
R^{\mu \nu}+\left(\lambda-\frac{1}{2} R\right) g^{\mu \nu}=-k\left(M^{\mu \nu}+S^{\mu \nu}\right)
$$

where $\lambda$ is the cosmological constant and $k$ is a constant, while $\left(M^{\mu \nu}\right)$ and $\left(S^{\mu \nu}\right)$ are the energy-momentum tensors of matter and the electromagnetic field, respectively. For incoherent matter (dust) $M^{\mu \nu}=\rho u^{\mu} u^{\nu}, \rho$ being the proper density and $\left(u^{\mu}\right)$ the 4-velocity $\left(c d x^{\mu} / d s\right), c$ being the speed of light in empty space.

Since the divergence of the left side of the set (Al) vanishes,

$$
-S_{; \nu}^{\mu \nu}=M_{; \nu}^{\mu \nu}=u^{\mu}\left(\rho u^{\nu}\right)_{; \nu}+\rho u^{\mu} ; \nu^{\nu \nu}
$$

It follows from $u_{\mu} u^{\mu}=c^{2}$ that $\left(u_{\mu} u^{\mu}\right)_{; \nu}$ and hence $u_{\mu} u^{\mu} ; \nu$ vanish. Also, the 4-force per unit proper volume $-\left(S^{\mu \nu} ; \nu\right)$ is orthogonal to $\left(u^{\mu}\right)$. So on multiplying through by $u_{\mu}$ and summing over $\mu$, (A2) leads to $\left(\rho u^{v}\right)_{j v}=0$. Substituting this into (A2) gives

$$
\rho u^{\nu} u_{; \nu}^{\mu}=-S^{\mu \nu} ; \nu
$$

- the equation of motion of the matter. The components of the 4-force per unit proper volume can be written as $\bar{\rho} \phi \mu_{u_{\alpha}}^{\alpha} / c, \bar{\rho}$ being the proper charge density. So (A3) can be written as equation (1) of the main text, with $a=-\bar{\rho} / \rho c$.

Using the general expression for a covariant derivative and $u^{\nu}=c d x^{\nu} / d s,(\mathrm{~A} 3)$ takes the alternative form 


$$
\rho\left[\frac{d^{2} x^{\mu}}{d s^{2}}+\left\{\begin{array}{c}
\mu \\
\alpha \beta
\end{array}\right\} \frac{d x^{\alpha}}{d s} \frac{d x^{\beta}}{d s}\right]=\rho \frac{\vec{\rho}}{\rho c^{3}} \phi^{\alpha \mu} u_{\alpha}
$$

where the braces denote Christoffel symbols of the second kind.

In the case of no non-gravitational fields, Sen $[15 ; 16, p .20]$ obtained the equation of motion of a single test particle of unit mass from that for dust by writing $\rho(x)=\delta(x-\bar{x}) / \sqrt{-g} ; \quad \delta$ is a four dimensional delta function, $x$ and $\bar{x}$ represent points in space-time and $g=\operatorname{det}\left(g_{\mu \nu}\right)$. His procedure can be generalized to the problem of a test charge in an electromagnetic field as follows. Substitute $\rho(x)=m \delta(x-\bar{x}) / \sqrt{-g}$ and $\bar{\rho}(x)=q \delta(x-\bar{x}) / \sqrt{-g}$ into (A4), $m$ and $q$ being the mass and charge of the particle. Multiplying through by $\sqrt{-g}$, integrating over all space-time and dropping the bars from $\bar{x}$ in the resulting equation gives

$$
\frac{d^{2} x^{\mu}}{d s^{2}}+\left\{\begin{array}{c}
\mu \\
\alpha \beta
\end{array}\right\} \frac{d x^{\alpha}}{d s} \frac{d x^{\beta}}{d s}=\frac{q}{m c^{3}} \phi^{\alpha \mu_{u_{\alpha}}}
$$

- the equation of motion of a test charge. Equation (A5) can also be written in the form of equation (1) of the main text, with a now equal to $-q / m c$.

\section{References}

[1] H. Bondi, "Relativity", Rep. Progr. Phys. 22 (1959), 97-120.

[2] S.N. Bose, "The affine connection in Einstein's new unitary field theory", Ann. of Math. (2) 59 (1954), 171-176.

[3] R. Burman, "Particle paths of general relativity as geodesics of an affine connection", Bull. Austral. Math. Soc. 3 (1970), 325-335.

[4] P. Droz-Vincent, "Electromagnetism and geodesics", Nuovo Cimento $B$ 51 (1967), 555-556.

[5] Albert Einstein, The meaning of relativity, 6th ed. (Methuen, London, 1956). 
[6] A. Einstein and B. Kaufman, "Algebraic properties of the field in the relativistic theory of the asymmetric field", Ann. of Math. (2) $59(1954), 230-244$.

[7] A. Einstein and B. Kaufman, "A new form of the general relativistic field equations", Ann. of Math. (2). 62 (1955), 128-138.

[8] Václav Hlavatý, "The elementary basic principles of the unified theory of relativity. B", J. Rational Mech. Anal. 2 (1953), $1-52$.

[9] Václav Hlavatý, "The elementary basic principles of the unified theory of relativity. $B_{2}$ ", J. Rational Mech. Anal. 4 (1955), $247-277$.

[10] Václav Hlavatý, "The elementary basic principles of the unified theory of relativity. $B_{3}$ ", J. Rational Mech. Anal. 4 (1955), 653-679.

[11] Václav Hlavaty, Geometry of Einstein's unified field theory (Noordhoff, Groningen, 1957).

[12] V. Hlavatý and A.W. Sáenz, "Uniqueness theorems in the unified theory of relativity", J. Rational Mech. Anal. 2 (1953), 523-536.

[13] B. Kaufman, "Mathematical structure of the non-symmetric field theory", Jubilee of relativity theory: Proceedings of a Conference held in Berne, July, 1955. Helv. Phys. Acta. Suppl. IV, 227-238. (Birkhäuser Verlag, Basel, 1956.)

[14] Erwin Schrödinger, Space-time stmucture (Cambridge University Press, Cambridge, 1950).

[15] D.K. Sen, "A simple derivation of the geodesic equations of motion from the matter tensor in general relativity using the S-function", Nuovo Cimento 21 (1961), 184-185.

[16] D.K. Sen, Fields and/or particles (The Ryerston Press, Toronto; Academic Press, London, New York, 1968).

[17] Marie-Antoinette Tonnelat, La théorie du chomp unifié d'Einstein et quelques-uns de ses dêveloppements (Gauthier-Villars, Paris, 1955). 
[18] Marie-Antoinette Tonnelat, "Les espoirs et les difficultés de la théorie du champ unifié d'Einstein", Recent developments in general relativity, 107-118. (Pergamon, Oxford; PWN-Polish Scientific Publishers, Warszawa, 1962).

University of Western Australia,

Nedlands,

Western Australia. 